\title{
Reciprocal Operation of Ultrasonic Transducers: Experimental Results
}

\author{
Johan Borg*, Jonny Johansson, Jan van Deventer and Jerker Delsing \\ EISLAB, Dept. of Computer Science and Electrical Engineering, \\ Luleå University of Technology, SE-971 87 Luleå, Sweden. \\ *Email: johan.borg@1tu.se
}

\begin{abstract}
Ultrasonic transit-time flow-meters estimate fluid or gas flows from the difference in times of flight of upstream and downstream acoustic pulses. However, any delay differences arising from sources other than the flow to be measured will cause a troublesome "zero flow" offset error.

In theory, the transducers used in the measurement system should not influence the zero flow error, as electroacoustic systems based on piezoelectric transducers have been shown to be reciprocal (when the media is stationary). However, care is required when designing the electrical interfaces for the piezoelectric transducers, if reciprocity in the system is to be utilized.

This work presents technique and measurements that apply reciprocity to an ultrasonic transit-time flow-meter. Specialized electrical transducer interfaces with options to drive the transducers from either low or high impedance sources were used. Combined with a high-impedance receive mode these options made it possible to change the conditions for reciprocity in the system.

We show reduced delay difference in 9 cases out of 10 when trying to utilize the reciprocal property compared to when we disregard it in favor for larger excitation energy. The delay improvements were accompanied by reduced differences between the center frequencies of the signals from the two paths.
\end{abstract}

\section{INTRODUCTION}

The common notion of reciprocity is the electromagnetic reciprocity theorem [1] developed by Henrik Lorentz during the late 19 th century as an extension of the older acoustic reciprocity formulated by Helmoltz. It applies to generic cases of linear time-invariant systems, but not without some exceptions, for example the ferromagnetic Faraday effect. When applied to circuit theory several simplifications can be made. A useful result is that given the current $I_{k}^{n}$ through voltage source $k$ when the only active voltage source $n$ is driving a voltage $V$, we have:

$$
I_{2}^{1}=I_{1}^{2}
$$

This can be formulated for arbitrary numbers of sources and can, by applying Norton and Tevenin equivalent circuits, be applied to current sources and ports with impedances.

Piezoelectric resonators are reciprocal in an electri$\mathrm{cal} /$ mechanical two-port sense [2] and a cascade of reciprocal networks is also reciprocal. Thus, any system with two piezoelectric transducers will constitute a reciprocal electrical twoport. An ultrasonic transit-time flow meter is essentially two piezoelectric transducers in an assembly where the fluid or gas will flow along the acoustic path between the transducers, either entirely along the same axis, as shown in Fig. 1, or at some non-normal angle. Reciprocity will naturally not hold in the case when the media between the transducers have a velocity component parallel to the acoustic signal, as the effective path for the different directions will be different, but it is still of considerable interest when trying to mitigate the measurement error when the flow is zero, the "zero flow" error [3].



Fig. 1. The flow-meter assembly (principle)

In this paper we have studied the measurable level of reciprocity expressed in terms of delay difference and "zero flow" accuracy, that is, the difference in transmission delay in both directions through a flow-meter assembly, and the corresponding flow, when the media is stationary. Using custom built electrical transducer interfaces we show improvements in the zero-flow error in 9 cases out of 10, and considerably reduced sensitivity to unequal capacitive loading. The presented work is a part of our ongoing research on integrated electronics for ultrasonic measurement systems [4], [5].

Section 2 presents the measurement system used, with experiments and results in sections 3 and 4, respectively. Discussion follows in section 5 with conclusions and future work in section 6 .

\section{EXPERIMENT SETUP}

Traditional interfaces for piezoelectric transceivers typically apply a voltage pulse without regard to the impedance load on the piezoelectric crystal during transmission [6]. Simulations of this kind of system has failed to show the expected reciprocal behavior [7]. In many cases, the crystal is significantly loaded either by the output transistor(s) or through the diodes that protect the receiver until the voltage across the crystal has stabilized. In contrast, in this paper, we attempt 
to drive the crystals from circuits designed to apply a current pulse and leave the crystal as lightly loaded as possible. The generation of short current pulses and the fact that the receive amplifier must be isolated from high voltages present some practical problems:

- Switching currents of $1 \mathrm{~A}$ (or more) fast enough is not trivial.

- The unavoidable series inductance between the driver and the transducer will result in a voltage drop when the current is switched on. This might force the discharging transistor to leave the saturation region, with reduced average output impedance as a result.

- When the current decreases rapidly at the end of the discharge, a high voltage is generated across the inductance until the stored energy has dissipated. At the current levels associated with driving the highly capacitive piezoelectric crystals, even very small inductances can store enough energy to damage an improperly designed interface.

- The switches used to isolate the receiver must withstand moderately high voltages and still have a low enough product of on resistance and parallel capacitance. Otherwise the variations in the $\mathrm{R}-\mathrm{C}$ time constant caused by the voltage and temperature dependence of the on resistance of the switch may degrade the delay stability significantly. For the same reason, the amplifier must have a high and/or very stable bandwidth.

The minimum practical length (about $2 \mathrm{~cm}$ ) of $0.635 \mathrm{~mm}$ pitch flat-cable (one pair) was used to connect the transducer and the interface, in order to minimize the series inductance. An ASIC from an earlier project is used as a very fast highvoltage transistor with an integrated driver [4]. The output transistor can withstand supply voltages of up to $40 \mathrm{~V}$ and the saturation current is $2 \mathrm{~A}$. However, because it is only capable of sinking, not sourcing, the piezoelectric crystal was first charged through an analog switch and then discharged through the ASIC after a long enough delay to allow all acoustic excitation from the charging stage to die out. The interface also contained a National Semiconductors LMH6702 current feedback OP-AMP used to amplify the signal and to drive the $50 \Omega$ output load. The switches in an Analog Devices ADG1213 are used to charge the crystal before the discharge and to isolate the amplifier during the discharge as shown in Fig. 2. PZ1 refers to the piezoelectric crystal in the transducer.

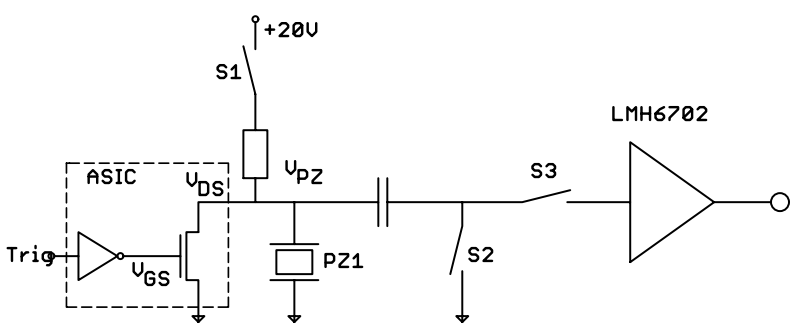

Fig. 2. Simplified schematic of the transducer interface
We distinguish between two modes of operation:

- The "Current mode" where the driving transistor is switched directly to and from operation in the saturation region, defined by $v_{P Z}=v_{D S}>v_{G S}-V_{t}$, where it acts as a current source in parallel with a moderately large resistor. In this application $v_{G S}-V_{t}$ is about $2.9 \mathrm{~V}$, which means the transistor must be turned off before the load has been discharged to this voltage. A discharge time of $20 \mathrm{~ns}$ was chosen for our application. See Fig. 3, the solid line.

- The "Voltage mode" where the driving transistor acts like a current source during the first part of the discharge, but stays on much longer allowing the voltage to drop below $2.9 \mathrm{~V}$ where the transistor starts acting like a small resistor instead. Fig. 3, dashed line.

It should be noted that the current mode not only utilizes the reciprocal properties, but also keeps the piezoelectric crystals operating in the parallel resonant mode during both the transmit and the receive phase, while the voltage mode drivers pulls the crystal towards series resonance during transmission, as the electrical terminals of the crystal are almost shorted.

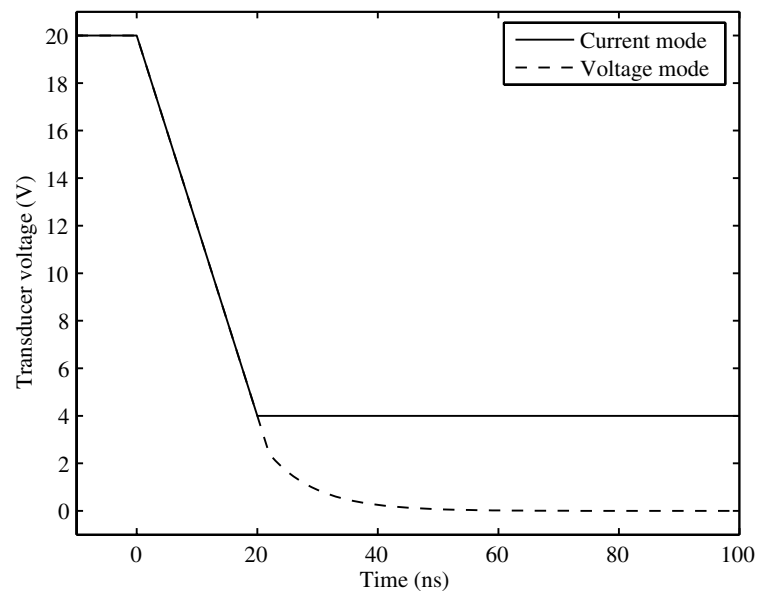

Fig. 3. Ideal discharge curves

The measurement setup was originally designed for mass flow measurements and is described in detail in [8]. It consists of two density probe ultrasonic transducers mounted in a custom assembly as schematically shown in Fig. 1. A measurement is initiated by sending a trig pulse to the interface electronics of both transducers simultaneously, initiating the discharge of energy stored in the piezoelectric crystals in the transducers. This will produce acoustic waves that travels to the opposite transducers where they are converted to electrical signals suitable for digitalization and further processing.

For this study, four new transducers were manufactured from 2 pairs of PZ-27 crystals with a diameter of $16 \mathrm{~mm}$ and a thickness of $0.96 \mathrm{~mm}$. The resonant frequencies before transducer assembly were $2190 \mathrm{kHz}$ and $2210 \mathrm{kHz}$, respectively for the pairs. The capacitance of the crystals measured well below the resonant frequency is about $3 \mathrm{nF}$. The transducers include 
TABLE I

MISMATCH FOR DIFFERENT TRANSDUCER COMBINATIONS

\begin{tabular}{lccccccccc}
\hline \multicolumn{4}{c|}{ Current mode } & \multicolumn{3}{c}{ Voltage mode } & \multicolumn{2}{c}{ Improvement } \\
\hline conf. & $\Delta t_{x}(\mathrm{~ns})$ & $\Delta t_{z}(\mathrm{~ns})$ & freq. (Hz) & $\Delta t_{x}(\mathrm{~ns})$ & $\Delta t_{z}(\mathrm{~ns})$ & freq. (Hz) & xcorr & zero crossing \\
\hline $3-4$ & -0.615 & -0.563 & 226.4 & 4.341 & 1.959 & -1356 & 7.05 & 3.48 \\
$3-1$ & -0.377 & -0.129 & 426.6 & -3.089 & -1.878 & 2092 & 8.20 & 14.5 \\
$3-2$ & 0.283 & 1.127 & 238.1 & 1.400 & -0.993 & 109.8 & 4.94 & 0.88 \\
$1-2$ & 0.317 & 0.390 & 31.03 & 3.409 & 2.731 & -1386 & 10.7 & 7.01 \\
$1-4$ & 1.579 & 1.321 & -446.6 & 5.460 & 3.418 & -3196 & 3.46 & 2.59 \\
\hline
\end{tabular}

a $50 \mathrm{~mm}$ length of polyetheretherketone (PEEK) polymer in front of the piezoelectric crystals in order to make the transducers usable in future density measurement applications. The backing consists of $9 \mathrm{~mm}$ of epoxy loaded with tungsten particles. This thickness was selected to make it possible to minimize the length of the electrical connections to the piezoelectric crystals while keeping any echo from the other side of the backing from interfering with the echos from the two reference surfaces.

\section{Measurements}

The outputs from the two transducers were connected to two channels of a Tektronix ${ }^{\mathrm{TM}}$ TDS7254 digital storage oscilloscope, used with a sampling frequency of $5 \mathrm{GHz}$ and set to average 64 waveforms before the data was transferred to a PC. Matlab ${ }^{\mathrm{TM}}$ was used for all digital signal processing.

Different delays in the isolating switches, amplifiers, connecting cables, etc, were canceled by performing all measurements twice and physically swapping the connections between the interfacing electronics and the transducers back and forth, as the purpose of the experiments was to measure the effects related to the transducers and of how they are driven.

The arrival time difference was calculated using two methods commonly used in the intended application: the delay associated with the highest cross correlation between the signals $\left(\Delta t_{x}\right)$ and the difference between the times of the zero crossing before the largest peak of each signal $\left(\Delta t_{z}\right)$. As seen in the results section, these methods do not, in general, give the same result in the presence of a frequency difference between the signals.

Two different experiments were performed to evaluate the use of "current mode" vs "voltage mode":

- The effects of different mismatch between the transducers were measured by mounting different combinations of our 4 transducers in the mass flow measuring assembly, whereafter the difference in arrival time of the signals from the two transducers was measured.

- Measurements were performed with a capacitive load placed across the electrical terminals of one of the transducers, in order to simulate the effects of severe mismatch between the transducers. These measurements can also be used to estimate how stable the capacitive loading of the transducers must be to avoid degrading the delay matching.
All experiments performed for this paper used stationary tap water in the mass flow meter assembly.

\section{RESULTS}

Table I shows the results when different combinations of transducers were used. "Improvement" is the absolute value of the delay difference in voltage mode divided by the corresponding delay difference measured when using current mode. "Frequency" shows the differences between the center frequencies of the received signals from the two transducers. In this flow-meter a delay difference of $1 \mathrm{~ns}$ corresponds to a flow of about $0.15 \mathrm{l} / \mathrm{min}$.

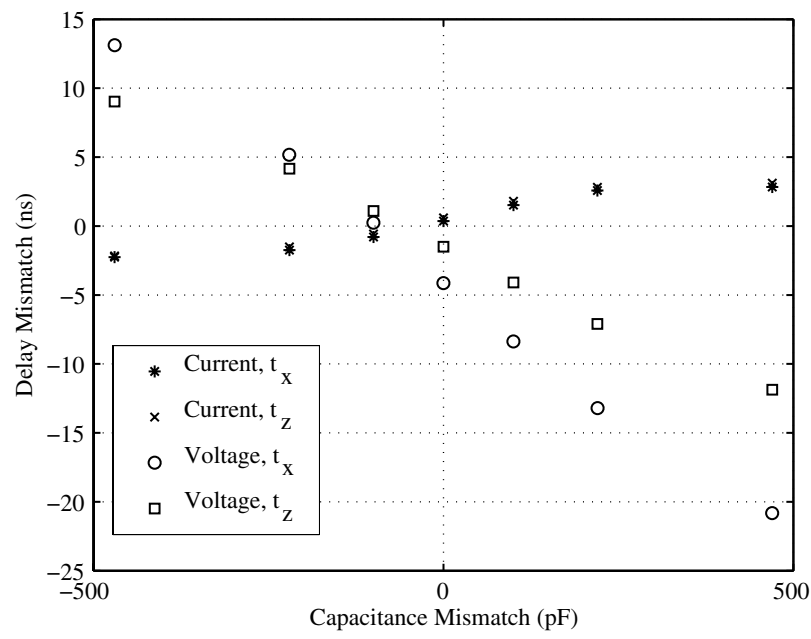

Fig. 4. Delay difference as a function of capacitive loading

Fig. 4 shows the results of loading the transducers with different capacitive loads, negative values on the $\mathrm{x}$-axis corresponds to loads at transducer A and positive values correspond to loads at transducer B. Fig. 5 shows the corresponding differences between the center frequencies of the signals from the two transducers.

The slopes of the curves were estimated by fitting lines with the least squares method. The calculated delay sensitivities are $6.1 \mathrm{ps} / \mathrm{pF}$ and $5.9 \mathrm{ps} / \mathrm{pF}$ in current mode and $34 \mathrm{ps} / \mathrm{pF}$ and $21 \mathrm{ps} / \mathrm{pF}$ in voltage mode for the values calculated using cross correlation and zero crossing, respectively. The corresponding values for the frequency sensitivities are $0.23 \mathrm{~Hz} / \mathrm{pF}$ and $9.9 \mathrm{~Hz} / \mathrm{pF}$, for current and voltage mode. 


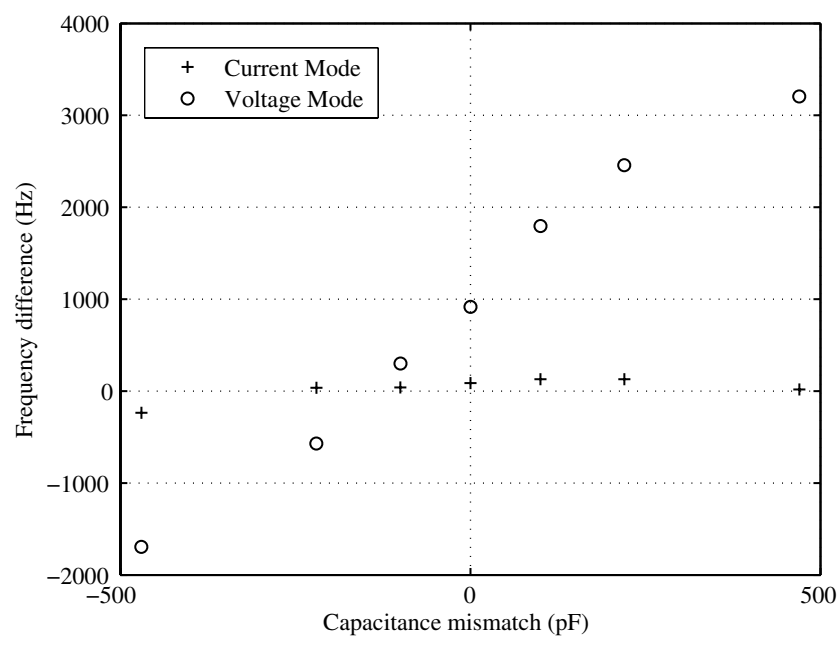

Fig. 5. Frequency difference as a function of capacitive loading

An estimate of the measurement stability was made through a series of measurements on a specific combination of transducers without additional loading, performed during the course of the main experiments. The data from these measurements provide estimated standard deviations of $0.18 \mathrm{~ns}$ and $0.3 \mathrm{~ns}$, for the values calculated using cross correlation and zero crossing, respectively.

\section{DISCUSSION}

We see a clear improvement in delay matching both when artificially loading a transducer (up to 7.5 times) and for most combinations of transducers (up to 14.5 times). It is interesting to note that the difference in frequency of the received pulses is reduced even more than the delay difference, 42 times compared to 5.5 times for our tests with artificially created mismatches.

However, the remaining delay difference measured when current mode was used is larger than expected.

We have identified a number of possible error sources:

- Different capacitive loading during transmission and reception, caused by the voltage dependent capacitance of the discharging transistor and the added capacitance when the receiving amplifier is connected. However, these capacitances are in the order of $10 \mathrm{pF}$, which should have negligible effects according to our experiments with asymmetric capacitive loading, even if the transducers are severely unmatched (for example, $100 \mathrm{pF}$ loading at $6 \%$ thickness mismatch).

- The finite output impedance of the discharging transistor which according to IC-simulations will be a few hundred ohms during discharge. It may be argued that this is a poor current source, but one should remember that this impedance is only present for $1 / 25$ th of the the oscillation period of the crystal. This makes the effective loading impedance several thousand ohms.
- The discharging transistor may leave saturation during some part of the discharge, either the start when any series inductances will interfere with the sudden switching of currents, or at the end, due to resistive voltage drops combined with the lower voltage over the transducer.

- The significantly larger improvements seen in the frequency difference versus the improvement of delay mismatch can possibly indicate a problem with the time of discharge rather than the reciprocal operation.

\section{CONCLUSIONS AND FUTURE WORK}

One way to utilize the reciprocal properties of an ultrasonic transit-time flow-meter system is to preserve high impedance across the transducer terminals in both transmit and receive phases. Experimental results show clear improvements in both transit time stability and frequency stability when current mode excitation is used, compared to voltage mode excitation. The transit time delay difference, and thus the zero-flow, is reduced for 9 test cases out of 10. For artificially created transducer mismatch the frequency stability improved a factor 42.

The findings will be used in the implementation of on-chip electronics for ultrasound systems. A more flexible driver with programmable current rise and fall times, as well as a high performance amplifier will be investigated. Isolation switches with on-resistance stabilization could be used to improve longterm stability, as could bandwidth stabilization of the amplifier.

\section{ACKNOWLEDGMENT}

The authors are thankful to Ferroperm A/S for kindly providing the piezoceramic discs for the transducers used in the experiments described in this paper. The work in this paper was funded by the European Union's action in support of regional development with Objective One.

\section{REFERENCES}

[1] S. Ramo, J. R. Whinnery, and T. V. Duzer, Fields and waves in communicatioin electronics, 3rd ed. John wiley \& sons inc, 1993, pp. 535-537.

[2] B. Auld, "Application of microwave concepts to the theory of acoustic fields and waves in solids," IEEE Transactions on Microwave Theory and Techniques, vol. MTT-17, no. 11, pp. 800 - 11, 1969/11/.

[3] J. Hemp, "Flowmeters and reciprocity," Quarterly Journal of Mechanics and Applied Mathematics, vol. 41, no. pt.4, pp. 503 - 20, 1988/11/.

[4] J. Johansson, M. Gustafsson, and J. Delsing, "Ultra-low power transmit/receive asic for battery operated ultrasound measurement systems," Sensors and Actuators, A: Physical, vol. 125, no. 2, pp. 317 - 328, 2006.

[5] J. Johansson and J. Delsing, "Microelectronics mounted on a piezoelectric transducer: Method, simulations, and measurements," Ultrasonics, vol. 44, no. 1, pp. $1-11,2006$.

[6] G. Hayward and M. N. Jackson, "Study of electronic switching devices for the characterisation of ultrasonic probe assemblies." Ultrasonics Symposium Proceedings, vol. 2, pp. 752 - 756, 1983.

[7] J. van Deventer and J. Delsing, "Apparent transducer non-reciprocity in an ultrasonic flow meter," Ultrasonics, vol. 40, no. 1-8, pp. $403-5$, 2002/05/.

[8] J. van Deventer, "Introduction of a 2 transducer ultrasonic mass flow meter," IMTC 2005. Proceedings of the 22th IEEE Instrumentation and Measurement Technology Conference., pp. 1369 - 1372, 2005. 